FACTA UNIVERSITATIS (NIŠ)

Ser. Math. Inform. Vol. 36, No 1 (2021), 125-141

https://doi.org/10.22190/FUMI200325011A

Original Scientific Paper

\title{
APPROXIMATION PROPERTIES OF MODIFIED BASKAKOV GAMMA OPERATORS
}

\author{
Seda Arpaguş and Ali Olgun
}

Kırıkkale University, Department of Mathematics, 71450 Kırıkkale, Turkey

\begin{abstract}
In this paper, we have studied an approximation properties of modified Baskakov-Gamma operator. Using Korovkin type theorem, firste we gave the approximation properties of this operator. Secondly, we computed the rate of convergence of this operator by means of the modulus of continuity and we gave an approximation properties of weighted spaces. Finally, we studied the Voronovskaya type theorem of this operator.
\end{abstract}

\section{Introduction}

The Baskakov operators and their connections with different branches of analysis such as convex and numerical analysis have been studied intensively.

In 1957, V.A. Baskakov defined the well _known Baskakov operators as follows[22];

$$
B_{n}(f ; x)=\sum_{k=0}^{\infty} f\left(\frac{k}{n}\right)\left(\begin{array}{c}
n+k-1 \\
k
\end{array}\right) \frac{x^{k}}{(1+x)^{n+k}} \quad x \geq 0, n \in \mathbb{N} .
$$

Later, many authors studied the approximation properties and gave many generalizations of these operators [1] ,[5],[10],[11],[15],[16],[17],[25],[26].Recently İnce İlarslan et al.[12] discussed some approximation properties of (p,q)-Baskakov-Kantoro -vich operators. Some authors studied the approximation properties Szasz type generalization[21].

Received March 25, 2020; accepted June 1, 2020.

Corresponding Author: Ali Olgun, Kırıkkale University, Department of Mathematics, 71450 Kırıkkale, Turkey | E-mail: aliolgun71@gmail.com

2010 Mathematics Subject Classification. Primary 41A10; Secondary 41A36, 41A25

(c) 2021 by University of Niš, Serbia | Creative Commons License: CC BY-NC-ND 
In 1998, V. Miheşan cosnructed and studied the convergence properties a generalization of the Baskakov operators as follows[24]:

$$
B_{n}^{a}(f ; x)=\sum_{k=0}^{\infty} e^{-\frac{a x}{1+x}} \frac{P_{k}(n, a)}{k !} \frac{x^{k}}{(1+x)^{n+k}} f\left(\frac{k}{n}\right) \quad x \geq 0, n \in \mathbb{N},
$$

where $P_{k}(x ; a)=\sum_{i=0}^{k}\left(\begin{array}{c}k \\ i\end{array}\right)(x)_{i} a^{k-i}$.

In [6], Wafi and Khatoon examined the convergence features of the integral type modification of the operators (1.1)

$$
V_{n}^{a}(f ; x)=n \sum_{k=0}^{\infty} e^{\frac{a x}{1+x}} \frac{P_{k}(n, a)}{k !} \frac{x^{k}}{(1+x)^{n+k}} \int_{\frac{k}{n}}^{\frac{k+1}{n}} f(t) d t \quad x \geq 0, n \in \mathbb{N}
$$

In 2010, Erençin and Başcanbaz-Tunca [2] identified a more general version of these operators, with the help of sequence, and examined the convergence features.

In 2011, Erençin constructed a Durrmeyer type modification of generalized Baskakov operators (1.1) as follows

$$
L_{n}^{\alpha}(f ; x)=e^{-\frac{a x}{1+x}} \sum_{k=0}^{\infty} \frac{P_{k}(n, a)}{k !} \frac{x^{k}}{(1+x)^{n+k}} \frac{1}{B(k+1, n)} \int_{0}^{\infty} \frac{t^{k}}{(1+t)^{n+k+1}} f(t) d t \quad ; \quad x \geq 0
$$

and studied some approximations properties[3].In (2012), Krech and Malejki investigated a modified type this operators[13].

In 2014, Erençin and Büyükdurakoğlu extended the operator (1.2) as

$$
K_{n}(f ; x)=e^{-\frac{a_{n} x}{1+x}} \sum_{k=0}^{\infty} \frac{P_{k}(n, a)}{k !} \frac{x^{k}}{(1+x)^{n+k}} \frac{b_{n}}{d_{n}-c_{n}} \int_{\frac{k+c_{n}}{b_{n}}}^{\frac{k+d_{n}}{b_{n}}} f(t) d t \quad ; x \geq 0, n \in \mathbb{N},
$$

which is a more general version of the operators and examined the convergence features in weighted spaces[1].

In 2017, N. Rao and A. Wafi [8] defined as follows

$$
L_{n, a}^{\alpha, \beta}(f, x)=e^{-\frac{a x}{1+x}} \sum_{k=0}^{\infty} \frac{P_{k}(n, a)}{k !} \frac{x^{k}}{(1+x)^{n+k}} f\left(\frac{k+\alpha}{n+\beta}\right)
$$

and examined the convergence features of Stancu variant the operator of (1.2) .

In 2015, Goyal and Agrawal examined the convergence features of bivariate generalization of operators $L_{n}^{\alpha}$ given by $(1.3)[15]$. 
Gamma operator is identified as[7]

$$
G_{n}(f, x)=\int_{0}^{\infty} \frac{x^{n+1}}{n !} e^{-x y} y^{n} f\left(\frac{n}{y}\right) d y \quad x \in(0, \infty), n \in \mathbb{N} .
$$

In 2011, L. Rempulska and M. Skorupka extended the modified version of Gamma operator as follows

$$
G_{n, p}(f, x)=\int_{0}^{\infty} \frac{x^{n+1}}{n !} e^{-x y} y^{n} F_{p}\left(x, \frac{n}{y}\right) d y
$$

and investigated the approximation properties for differentiable functions in polynomial weighted spaces[14].

Different modification of this operator were examined[19],[20],[21].

In 2014, R. Malejki and E. Wachnicki[18] constructed integral type modification the operators $B_{n}^{\alpha}$ given by(1.1)as follows:

$$
M_{n}^{\alpha, a}(f, x)=e^{-\frac{a x}{1+x}} \sum_{k=0}^{\infty} \frac{P_{k}(n, a)}{k !} \frac{x^{k}}{(1+x)^{n+k}} \frac{1}{\Gamma(\alpha+k+1)} \int_{0}^{\infty} e^{-n s}(n s)^{\alpha+k} f(s) d s
$$

and studied approximation properties of the operator. In (2015), E. Pandey and S.P. Mishra investigated a differet type this operators [9].

In 2016, I. Krech and R. Malejki[13] defined a multivariate version of the operators $M_{n}^{\alpha, a}$.

In this paper, we give a new generalization consisting of the linear combination of Baskakov- Gamma operators.

\section{Constructions of the Operators}

Let $x \in(0, \infty), n \in \mathbb{N}, 0<\alpha<\beta$ and $f$ be defined on the space $C_{B}(0, \infty)$ of all continuous bounded functions. We define the operator as follows:

$$
S_{n, a}^{\alpha, \beta}(f ; x)=e^{-\frac{a x}{1+x}} \sum_{k=0}^{\infty} \frac{P_{k}(n, a)}{k !} \frac{x^{k}}{(1+x)^{n+k}} \int_{0}^{\infty} \frac{x^{n+1}}{n !} y^{n} e^{-x y} f\left(\frac{\frac{k n}{x y}+\alpha}{n+\beta}\right) d y
$$

where $a>0$ is a constant and

$$
P_{k}(n, a)=\sum_{i=0}^{k}\left(\begin{array}{l}
k \\
i
\end{array}\right)(n)_{i} a^{k-i}
$$


with $(n)_{0}=1,(n)_{i}=n(n+1)(n+2) \ldots(n+i-1) ; i \geq 1$ denotes Pochammer Symbol. With the help of derivatives, $e^{-\frac{a x}{1+x}} \sum_{k=0}^{\infty} \frac{P_{k+1}(n, a)}{k !} \frac{x^{k}}{(1+x)^{n+k}}=n(1+x)+a$ and $e^{-\frac{a x}{1+x}} \sum_{k=0}^{\infty} \frac{P_{k+2}(n, a)}{k !} \frac{x^{k}}{(1+x)^{n+k}}=n(n+1)(1+x)^{2}+2 a n(1+x)+a^{2}$ can be easily proved.

\section{Auxiliary results}

Lemma 3.1. For the operators (2.1), we have

$$
\begin{aligned}
S_{n, a}^{\alpha, \beta}(1 ; x) & =1, \\
S_{n, a}^{\alpha, \beta}(t ; x) & =\frac{n x+\alpha}{n+\beta} \frac{a x}{(1+x)(n+\beta)}, \\
S_{n, a}^{\alpha, \beta}\left(t^{2} ; x\right) & =\frac{n^{2}(1+n) x^{2}}{(n+\beta)^{2}(n-1)}+\frac{\left[2 a n^{2}(1+x)+\alpha^{2} n\right]}{(n+\beta)^{2}(n-1)} \frac{x^{2}}{(1+x)^{2}} \\
& \times \frac{\left\{\left[n^{2}+2 \alpha n(n-1)\right](1+x)+[a n+2 a \alpha(n-1)]\right\}}{(n+\beta)^{2}(n-1)} \frac{x}{1+x}+\frac{\alpha^{2}}{(n+\beta)^{2}} .
\end{aligned}
$$

Proof. Using the operator (2.1), , it follows

$$
S_{n, a}^{\alpha, \beta}(1 ; x)=e^{-\frac{a x}{1+x}} \sum_{k=0}^{\infty} \frac{P_{k}(n, a)}{k !} \frac{x^{k}}{(1+x)^{n+k}} \frac{x^{n+1}}{n !} \int_{0}^{\infty} y^{n} e^{-x y} d y
$$

If we say $x y=t$ then it follows

$$
S_{n, a}^{\alpha, \beta}(1 ; x)=e^{-\frac{a x}{1+x}} \sum_{k=0}^{\infty} \frac{P_{k}(n, a)}{k !} \frac{x^{k}}{(1+x)^{n+k}}=1,
$$

which proves the first result.

For $f(t)=t$ we have

$$
\begin{aligned}
& S_{n, a}^{\alpha, \beta}(t ; x) \\
& =e^{-\frac{a x}{1+x}} \sum_{k=0}^{\infty} \frac{P_{k}(n, a)}{k !} \frac{x^{k}}{(1+x)^{n+k}} \frac{x^{n+1}}{n !} \frac{1}{n+\beta}\left[n \int_{0}^{\infty} y^{n} e^{-x y} \frac{k}{x y} d y+\alpha \int_{0}^{\infty} y^{n} e^{-x y} d y\right] \\
& =\frac{1}{n+\beta} \frac{x}{1+x}\left(e^{-\frac{a x}{1+x}} \sum_{k=0}^{\infty} \frac{P_{k+1}(n, a)}{k !} \frac{x^{k}}{(1+x)^{n+k}}\right)+\frac{\alpha}{n+\beta}=\frac{n x+\alpha}{n+\beta}+\frac{a x}{(1+x)(n+\beta)}
\end{aligned}
$$

For $f(t)=t^{2}$, it follows

$$
S_{n, a}^{\alpha, \beta}\left(t^{2} ; x\right)=e^{-\frac{a x}{1+x}} \sum_{k=0}^{\infty} \frac{P_{k}(n, a)}{k !} \frac{x^{k}}{(1+x)^{n+k}} \frac{x^{n+1}}{n !} \int_{0}^{\infty} y^{n} e^{-x y}\left(\frac{\frac{k n}{x y}+\alpha}{n+\beta}\right)^{2} d y
$$




$$
\begin{aligned}
& =\frac{e^{-\frac{a x}{1+x}}}{(n+\beta)^{2}} \sum_{k=0}^{\infty} \frac{P_{k}(n, a)}{k !} \frac{x^{k+n+1}}{(1+x)^{n+k} n !} \\
& \times\left[n^{2} \int_{0}^{\infty} y^{n} e^{-x y} \frac{k^{2}}{x^{2} y^{2}} d y+2 \alpha n \int_{0}^{\infty} y^{n} e^{-x y} \frac{k}{x y} d y+\alpha^{2} \int_{0}^{\infty} y^{n} e^{-x y} d y\right] \\
& =\frac{n}{(n-1)(n+\beta)^{2}} \frac{x^{2}}{(1+x)^{2}}\left(e^{-\frac{a x}{1+x}} \sum_{k=0}^{\infty} \frac{P_{k+2}(n, a)}{k !} \frac{x^{k}}{(1+x)^{n+k}}\right) \\
& +\frac{n}{(n-1)(n+\beta)^{2}} \frac{x}{1+x} \times\left(e^{-\frac{a x}{1+x}} \sum_{k=0}^{\infty} \frac{P_{k+1}(n, a)}{k !} \frac{x^{k}}{(1+x)^{n+k}}\right) \\
& +\frac{2 \alpha}{(n+\beta)^{2}} \frac{x}{1+x}\left(e^{-\frac{a x}{1+x}} \sum_{k=0}^{\infty} \frac{P_{k+1}(n, a)}{k !} \frac{x^{k}}{(1+x)^{n+k}}\right)+\frac{\alpha^{2}}{(n+\beta)^{2}} \\
& =\frac{n^{2}(1+n) x^{2}}{(n+\beta)^{2}(n-1)}+\frac{\left[2 a n^{2}(1+x)+\alpha^{2} n\right]}{(n+\beta)^{2}(n-1)} \frac{x^{2}}{(1+x)^{2}} \\
& +\frac{\left\{\left[n^{2}+2 \alpha n(n-1)\right](1+x)+[a n+2 a \alpha(n-1)]\right\}}{(n+\beta)^{2}(n-1)} \frac{x}{1+x}+\frac{\alpha^{2}}{(n+\beta)^{2}},
\end{aligned}
$$

which completes the proof.

$S_{n, a}^{\alpha, \beta}\left(t^{3} ; x\right)$ and $S_{n}^{a}\left(t^{4} ; x\right)$ can be proved in a similarly way that of the proof of Lemma 3.1.

Theorem 3.1. Let $f \in C_{B}(0, \infty), x \in(0, \infty)$ and $n \in \mathbb{N}$. Then we have

$$
\lim _{n \rightarrow \infty}\left(S_{n, a}^{\alpha, \beta}(f ; x)-f(x)\right)=0 .
$$

Proof. Proof is clear that by Lemma 3.1 .

Lemma 3.2. For the operators (2.1),

$$
S_{n, a}^{\alpha, \beta}\left((t-x)^{2} ; x\right) \leq M^{*} \frac{x^{2}+x+1}{(n+\beta)^{2}}
$$

where $M_{i}=(n, a, \beta, \alpha), i=1,2, \ldots ; \quad M^{*}=\max \left(M_{i}\right)$.

Proof. From linearity of the operator $(2.1)$ and Lemma3.1, since $\frac{x^{s}}{(1+x)^{l}} \leqq x^{s}$ for all $x \geqq 0, \quad l<s \quad(l, s=1,2,3,4)$, we can write

$$
\begin{aligned}
S_{n, a}^{\alpha, \beta}\left((t-x)^{2} ; x\right) & \leq \frac{1}{(n+\beta)^{2}}\left(\frac{n\left(n+\alpha^{2}+2 a n+n^{2}\right)-(n-1)(n+\beta)(n+2 \alpha-\beta)}{n-1}\right) x^{2} \\
& +\frac{1}{(n+\beta)^{2}}\left(\frac{(n-2 \alpha+2 n \alpha)(a+n)-2 \alpha(n-1)(n+\beta)}{n-1}\right) x+\frac{\alpha^{2}}{(n+\beta)^{2}} \\
& =\frac{x^{2}}{(n+\beta)^{2}} M_{1}+\frac{x}{(n+\beta)^{2}} M_{2}+\frac{1}{(n+\beta)^{2}} M_{3} \\
& \leq M^{*} \frac{x^{2}+x+1}{(n+\beta)^{2}} .
\end{aligned}
$$




\section{Rates of Convergence}

We can show the approximation of the operator with the help of the modulus of continuity.

Theorem 4.1. Let $x \in(0, \infty), n \in \mathbb{N}$ and $f \in C_{B}$, then we have

$$
\left|S_{n, a}^{\alpha, \beta}(f ; x)-f(x)\right| \leq M^{* *} w\left(f ; \sqrt{\frac{x^{2}+x+1}{(n+\beta)^{2}}}\right) .
$$

Proof. By the definition of the operators (2.1) and properties of modulus of continuity, we may write

$$
\begin{aligned}
& \left|S_{n, a}^{\alpha, \beta}(f ; x)-f(x)\right| \leq \\
\leq & e^{-\frac{a x}{1+x}} \sum_{k=0}^{\infty} \frac{P_{k}(n, a)}{k !} \frac{x^{k}}{(1+x)^{n+k}} \frac{x^{n+1}}{n !} \int_{0}^{\infty} y^{n} e^{-x y}\left|f\left(\frac{\frac{k n}{x y}+\alpha}{n+\beta}\right)-f(x)\right| d y \\
\leq & e^{-\frac{a x}{1+x}} \sum_{k=0}^{\infty} \frac{P_{k}(n, a)}{k !} \frac{x^{k}}{(1+x)^{n+k}} \frac{x^{n+1}}{n !} \int_{0}^{\infty} y^{n} e^{-x y} w\left(f ;\left|\frac{\frac{k n}{x y}+\alpha}{n+\beta}-x\right|\right) d y \\
= & w(f, \delta)+e^{-\frac{a x}{1+x}} \sum_{k=0}^{\infty} \frac{P_{k}(n, a)}{k !} \frac{x^{k}}{(1+x)^{n+k}} \frac{x^{n+1}}{n !} \frac{1}{\delta} w(f ; \delta)\left(\int_{0}^{\infty} y^{n} e^{-x y}\left|\frac{\frac{k n}{x y}+\alpha}{n+\beta}-x\right| d y\right)
\end{aligned}
$$

By applying the Cauchy-Schwarz inequality two times succesively to the right side, we get

$$
\left|S_{n, a}^{\alpha, \beta}(f ; x)-f(x)\right| \leq w(f, \delta)
$$

$$
\begin{aligned}
& +\frac{1}{\delta} w(f ; \delta)\left\{\left(e^{-\frac{a x}{1+x}} \sum_{k=0}^{\infty} \frac{P_{k}(n, a)}{k !} \frac{x^{k}}{(1+x)^{n+k}} \int_{0}^{\infty} \frac{x^{n+1}}{n !} y^{n} e^{-x y}\left(\frac{\frac{k n}{x y}+\alpha}{n+\beta}-x\right)^{2} d y\right)^{\frac{1}{2}}\right. \\
& \left.\times\left(e^{-\frac{a x}{1+x}} \sum_{k=0}^{\infty} \frac{P_{k}(n, a)}{k !} \frac{x^{k}}{(1+x)^{n+k}} \int_{0}^{\infty} \frac{x^{n+1}}{n !} y^{n} e^{-x y} d y\right)^{\frac{1}{2}}\right\} \\
& \leq w(f, \delta)+\frac{1}{\delta} w(f ; \delta) \sqrt{M^{*}} \sqrt{\frac{x^{2}+x+1}{(n+\beta)^{2}}}
\end{aligned}
$$

If we take $\delta=\sqrt{\frac{x^{2}+x+1}{(n+\beta)^{2}}}$, then it follows

$$
\left|S_{n, a}^{\alpha, \beta}(f ; x)-f(x)\right| \leq M^{* *} w\left(f ; \sqrt{\frac{x^{2}+x+1}{(n+\beta)^{2}}}\right) .
$$

which ends the proof where

$$
M^{* *}=1+\sqrt{M^{*}} .
$$


Let $C_{B}(0, \infty)$ denote the space of real valued continuous and bounded functions on the interval $(0, \infty)$, with the norm

$$
\|f\|=\sup _{0 \leq x<\infty}|f(x)| .
$$

For every $\delta>0$, Peetre's K- functional is defined by

$$
K_{2}(f ; \delta)=\inf _{g \in C_{B}^{2}(0, \infty)}\left\{\|f-g\|+\delta\left\|g^{\prime \prime}\right\|\right\}
$$

where

$$
C_{B}^{2}(0, \infty)=\left\{g \in C_{B}(0, \infty): g^{\prime}, g^{\prime \prime} \in C_{B}(0, \infty)\right\} .
$$

There exists an absolute constant $C>0$ such that

$$
K_{2}(f ; \delta) \leq C w_{2}(f ; \sqrt{\delta})
$$

holds where $w_{2}$ is the second order modulus of smoothness of $f$, defined by

$$
w(f ; \delta)=\sup _{0<h \leq \delta 0<x<\infty} \sup _{0<0}|f(x+2 h)-2 f(x+h)+f(x)| .
$$

Now, we consider the following $\hat{S}_{n, a}^{\alpha, \beta}(f ; x)$ by means of operator $S_{n, a}^{\alpha, \beta}$

$$
\hat{S}_{n, a}^{\alpha, \beta}(f ; x)=S_{n, a}^{\alpha, \beta}(f ; x)-f\left(\frac{a x+(1+x)(n x+\alpha)}{(1+x)(n+\beta)}\right)+f(x) .
$$

Then, the following Lemma can be given.

Lemma 4.1. Let $g \in C_{B}^{2}(0, \infty)$. Then we have

$$
\left|\hat{S}_{n, a}^{\alpha, \beta}(g ; x)-g(x)\right| \leq \delta_{n}(x)\left\|g^{\prime \prime}\right\|
$$

where

$$
\delta_{n}(x)=S_{n, a}^{\alpha, \beta}\left((t-x)^{2} ; x\right)+\left(\frac{a x+(1+x)(\alpha-x \beta)}{(1+x)(n+\beta)}\right)^{2} .
$$

Proof. For the operators $\hat{S}_{n, a}^{\alpha, \beta}(f ; x)$, we get

$$
\begin{aligned}
\hat{S}_{n, a}^{\alpha, \beta}(t-x ; x) & =S_{n, a}^{\alpha, \beta}(t-x ; x)-\left(\frac{a x+(1+x)(\alpha-x \beta)}{(1+x)(n+\beta)}\right) \\
& =S_{n, a}^{\alpha, \beta}(t ; x)-x S_{n, a}^{\alpha, \beta}(1 ; x)-S_{n, a}^{\alpha, \beta}(t ; x)+x S_{n, a}^{\alpha, \beta}(1 ; x)=0 .
\end{aligned}
$$


Let $g \in C_{B}^{2}(0, \infty)$ and $x \in(0, \infty)$. By Taylor's formula of $g$, we may write

$$
g(t)-g(x)=(t-x) g^{\prime}(x)+\int_{x}^{t}(t-u) g^{\prime \prime}(u) d u \quad ; \quad t \in[0, \infty) .
$$

If we apply the operator $\hat{S}_{n, a}^{\alpha, \beta}$ to this equality, we obtain

$$
\begin{aligned}
\hat{S}_{n, a}^{\alpha, \beta}(g(t)- & g(x) ; x)=g^{\prime}(x) \hat{S}_{n, a}^{\alpha, \beta}((t-x) ; x)+\hat{S}_{n, a}^{\alpha, \beta}\left(\int_{x}^{t}(t-x) g^{\prime \prime}(u) d u ; x\right) \\
= & \hat{S}_{n, a}^{\alpha, \beta}\left(\int_{x}^{t}(t-x) g^{\prime \prime}(u) d u ; x\right) \\
= & S_{n, a}^{\alpha, \beta}\left(\int_{x}^{t}(t-x) g^{\prime \prime}(u) d u ; x\right)- \\
& -\left(\int_{x}^{\frac{a x+(1+x)(n x+\alpha)}{(1+x)(n+\beta)}}\left(\frac{a x+(1+x)(n x+\alpha)}{(1+x)(n+\beta)}-u\right) g^{\prime \prime}(u) d u ; x\right) \\
& \quad+\int_{x}^{x}(x-u) d u .
\end{aligned}
$$

By using the following inequality

$$
\left|\int_{x}^{t}(t-u) g^{\prime \prime}(u) d u\right| \leq(t-x)^{2}\left\|g^{\prime \prime}(u)\right\|
$$

we can write

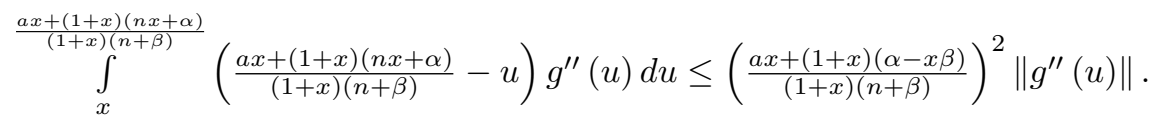

In wiev of this inequality, we can conclude that

$$
\begin{aligned}
\left|\hat{S}_{n, a}^{\alpha, \beta}(g ; x)-g(x)\right| & \leq\left\{S_{n, a}^{\alpha, \beta}\left((t-x)^{2} ; x\right)+\left(\frac{a x+(1+x)(\alpha-x \beta)}{(1+x)(n+\beta)}\right)^{2}\right\}\left\|g^{\prime \prime}\right\| \\
& =\delta_{n}(x)\left\|g^{\prime \prime}\right\| .
\end{aligned}
$$

Theorem 4.2. Let $f \in C_{B}(0, \infty)$. For all $x \in(0, \infty)$, there exists a constant $B>0$ such that

$$
\left|S_{n, a}^{\alpha, \beta}(f ; x)-f(x)\right| \leq B w_{2}\left(f ; \sqrt{\delta_{n}(x)}\right)+w\left(f ; \frac{a x+(1+x)(\alpha-x \beta)}{(1+x)(n+\beta)}\right)
$$


where

$$
\delta_{n}(x)=S_{n, a}^{\alpha, \beta}\left((t-x)^{2} ; x\right)+\left(\frac{a x+(1+x)(\alpha-x \beta)}{(1+x)(n+\beta)}\right)^{2}
$$

Proof. For the operators $\hat{S}_{n, a}^{\alpha, \beta}$, we write

$$
\hat{S}_{n, a}^{\alpha, \beta}(f ; x)-f(x)=\hat{S}_{n, a}^{\alpha, \beta}(f-g ; x)+(f-g)(x)+\hat{S}_{n, a}^{\alpha, \beta}(g-g(x) ; x)
$$

from the equality(4.1), it follows

$$
\begin{array}{r}
S_{n, a}^{\alpha, \beta}(f ; x)-f\left(\frac{a x+(1+x)(n x+\alpha)}{(1+x)(n+\beta)}\right)+f(x)-f(x)=\hat{S}_{n, a}^{\alpha, \beta}(f-g ; x)+(f-g)(x) \\
+\hat{S}_{n, a}^{\alpha, \beta}(g ; x)-g(x)
\end{array}
$$

and

$$
\begin{aligned}
\left|S_{n, a}^{\alpha, \beta}(f ; x)-f(x)\right| \leq \mid & \hat{S}_{n, a}^{\alpha, \beta}(f-g ; x)|+|(f-g)(x) \mid \\
& +\left|\hat{S}_{n, a}^{\alpha, \beta}(g ; x)-g(x)\right|+\left|f\left(\frac{a x+(1+x)(n x+\alpha)}{(1+x)(n+\beta)}\right)-f(x)\right| .
\end{aligned}
$$

By taking the supremum of $\hat{S}_{n, a}^{\alpha, \beta}$ operators, we get

$$
\begin{aligned}
& \left|\hat{S}_{n, a}^{\alpha, \beta}(f ; x)\right|=\left|S_{n, a}^{\alpha, \beta}(f ; x)-f\left(\frac{a x+(1+x)(n x+\alpha)}{(1+x)(n+\beta)}\right)+f(x)\right| \\
& \leq\left|S_{n, a}^{\alpha, \beta}(f ; x)\right|+2\|f\| \\
& \leq 3\|f\| \text {. }
\end{aligned}
$$

Now if equality (4.3) is replaced by inequality (4.4), we have

$$
\begin{aligned}
\left|S_{n}^{a}(f ; x)-f(x)\right| \leq & 4\|f-g\|+\left|\hat{S}_{n}^{a}(g ; x)-g(x)\right| \\
& +\left|f\left(\frac{a x+(1+x)(n x+\alpha)}{(1+x)(n+\beta)}\right)-f(x)\right|
\end{aligned}
$$

from Lemma4.1 we obtain

$$
\begin{aligned}
\left|S_{n, a}^{\alpha, \beta}(f ; x)-f(x)\right| \leq & 4\left\{\|f-g\|+\delta_{n}(x)\left\|g^{\prime \prime}\right\|\right\} \\
& +w\left(f ; \frac{a x+(1+x)(\alpha-x \beta)}{(1+x)(n+\beta)}\right) .
\end{aligned}
$$


By taking the infimum for all $g \in C_{B}^{2}(0, \infty)$ on the right-hand side of the last inequality and considering (4.1), we get that

$$
\begin{aligned}
\left|S_{n, a}^{\alpha, \beta}(f ; x)-f(x)\right| & \leq 4 K_{2}\left(f ; \delta_{n}\right)+w\left(f ; \frac{a x+(1+x)(\alpha-x \beta)}{(1+x)(n+\beta)}\right) \\
& \leq 4 C w_{2}\left(f ; \sqrt{\delta_{n}}\right)+w\left(f ; \frac{a x+(1+x)(\alpha-x \beta)}{(1+x)(n+\beta)}\right) \\
& =B w_{2}\left(f ; \sqrt{\delta_{n}}\right)+w\left(f ; \frac{a x+(1+x)(\alpha-x \beta)}{(1+x)(n+\beta)}\right)
\end{aligned}
$$

which completes the proof.

Theorem 4.3. Let $0<\gamma \leq 1$ and $f \in C_{B}(0, \infty)$. Then if $f \in \operatorname{Lip}_{M}(\gamma)$, that is, the inequality

$$
|f(t)-f(x)| \leq M|t-x|^{\gamma}, \quad x, t \in(0, \infty)
$$

holds, then for each $x \in(0, \infty)$ we have

$$
\left|S_{n, a}^{\alpha, \beta}(f ; x)-f(x)\right| \leq M \delta_{n}^{\frac{\gamma}{2}}(x)
$$

where

$\delta_{n}=S_{n, a}^{\alpha, \beta}\left((t-x)^{2} ; x\right)$ and $M>0$ is a constant.

Proof. Let $f \in C_{B}(0, \infty) \cap \operatorname{Lip}_{M}(\gamma)$. By the linearity and monotonicity of the $S_{n, a}^{\alpha, \beta}$ operators, we get

$$
\begin{aligned}
& \left|S_{n, a}^{\alpha, \beta}(f ; x)-f(x)\right| \leq S_{n, a}^{\alpha, \beta}(|f(t)-f(x)| ; x) \\
& \leq M S_{n, a}^{\alpha, \beta}\left(|t-x|^{\gamma} ; x\right) \\
& =M \sum_{k=0}^{\infty} e^{-\frac{a x}{1+x}} \frac{P_{k}(n, a)}{k !} \frac{x^{k}}{(1+x)^{n+k}} \frac{x^{n+1}}{n !} \int_{0}^{\infty} y^{n} e^{-x y}\left|\frac{\frac{k n}{x y}+\alpha}{n+\beta}-x\right|^{\gamma} d y \text {. }
\end{aligned}
$$

By applying the Hölder inequality two times succesively to the right side with $p=\frac{2}{\gamma}, q=\frac{2}{2-\gamma}$, we obtain

$$
\begin{aligned}
& \left|S_{n, a}^{\alpha, \beta}(f ; x)-f(x)\right| \\
\leq & M\left(e^{-\frac{a x}{1+x}} \sum_{k=0}^{\infty} \frac{P_{k}(n, a)}{k !} \frac{x^{k}}{(1+x)^{n+k}} \frac{x^{n+1}}{n !} \int_{0}^{\infty} y^{n} e^{-x y}\left|\frac{\frac{k n}{x y}+\alpha}{n+\beta}-x\right|^{2} d y\right)^{\frac{\gamma}{2}} \\
\leq & M S_{n, a}^{\alpha, \beta}\left((t-x)^{2} ; x\right)^{\frac{\gamma}{2}} \\
= & M \delta_{n}^{\frac{\gamma}{2}}(x),
\end{aligned}
$$

which is the desired result. 


\section{Weighted Approximation Properties}

Firstly, we give some definitions and theorem

Let $\rho(x)=1+x^{2}$ and $B_{\rho}[0, \infty)$ denote the space of all functions having the property

$$
|f(x)| \leq M_{f} \rho(x)
$$

where $x \in[0, \infty)$ and $M_{f}$ is a positive constant on $f$ functions. The norm on $B_{\rho}[0, \infty)$ is defined as follows

$$
\|f\|_{\rho}=\sup _{0 \leq x<\infty} \frac{|f(x)|}{1+x^{2}} .
$$

$C_{\rho}[0, \infty)$ denotes the space of all continuous functions belonging to $B_{\rho}[0, \infty)$ and $C_{\rho}^{0}[0, \infty)$ denotes the subspace of all functions $f \in C_{\rho}[0, \infty)$ for which

$$
\lim _{x \rightarrow \infty} \frac{|f(x)|}{\rho(x)}=0 .
$$

The basic theorem for approximation of weighted spaces is given by Gadjiev $\operatorname{in}[4]$.

Theorem 5.1. Let $\left\{A_{n}\right\}$ be a sequence of positive linear operators defined from $C_{\rho}^{0}[0, \infty)$ to $B_{\rho}[0, \infty)$, and satisfying the conditions

$$
\lim _{n \rightarrow \infty}\left\|A_{n}\left(t^{v} ; x\right)-x^{v}\right\|_{\rho}=0, \quad v=0,1,2 .
$$

Then for any $f \in C_{\rho}^{0}[0, \infty)$,

$$
\lim _{n \rightarrow \infty}\left\|A_{n}(f ; x)-f(x)\right\|_{\rho}=0 .
$$

It is shown in [4] that, a sequence of linear positive operators $A_{n}$ is defined from $C_{\rho}^{0}[0, \infty)$ to $B_{\rho}[0, \infty)$ if and only if

$$
\left\|A_{n}(\rho ; x)\right\|_{\rho} \leq M_{\rho}
$$

where $M_{\rho}$ is a positive constant.

Theorem 5.2. Let $\left\{S_{n, a}^{\alpha, \beta}\right\}$ be the sequence of positive linear operators. For each $f \in C_{\rho}^{0}(0, \infty)$, we have

$$
\lim _{n \rightarrow \infty}\left\|S_{n, a}^{\alpha, \beta}(f ; x)-f(x)\right\|_{\rho}=0 .
$$


Proof. Using Lemma3.2, we get

$$
\begin{aligned}
\sup _{0 \leq x<\infty} \frac{\left|S_{n, a}^{\alpha, \beta}(\rho ; x)\right|}{1+x^{2}} & =\sup _{0 \leq x<\infty} \frac{\left|S_{n, a}^{\alpha, \beta}\left(1+t^{2} ; x\right)\right|}{1+x^{2}} \\
& \leq 1+\frac{n\left(a+2 n+\alpha^{2}+2 a n+n^{2}\right)+\alpha(n-1)(2 a+2 n+\alpha)}{(n+\beta)^{2}(n-1)} .
\end{aligned}
$$

There exists a positive contant $D$ such that for each $n$ and $\alpha, a, \beta<\infty$

$$
\frac{n\left(a+2 n+\alpha^{2}+2 a n+n^{2}\right)+\alpha(n-1)(2 a+2 n+\alpha)}{(n+\beta)^{2}(n-1)}<D .
$$

Hence we may write

$$
\sup _{0 \leq x<\infty} \frac{\left|S_{n, a}^{\alpha, \beta}(\rho ; x)\right|}{1+x^{2}}=\left\|S_{n, a}^{\alpha, \beta}(\rho ; x)\right\|_{\rho} \leq 1+D .
$$

which shows that $\left\{S_{n}^{a}\right\}$ is a sequence of positive linear operators defined from $C_{\rho}^{0}(0, \infty)$ to $B_{\rho}(0, \infty)$.

For $v=0$, it is clear that

$$
\left\|S_{n, a}^{\alpha, \beta}(1 ; x)-1\right\|_{\rho}=0
$$

For $v=1$, we have

$$
\begin{aligned}
\left\|S_{n, a}^{\alpha, \beta}(t ; x)-x\right\|_{\rho} & =\sup _{0 \leq x<\infty} \frac{\left|S_{n, a}^{\alpha, \beta}(t ; x)-x\right|}{1+x^{2}} \\
& =\sup _{0 \leq x<\infty}\left|\frac{a x+(1+x)(n x+\alpha)}{(1+x)(n+\beta)} \frac{1}{1+x^{2}}-\frac{x}{1+x^{2}}\right| \\
& \leq\left|\frac{A}{n+\beta}\right|
\end{aligned}
$$

holds. Similarly, for $v=2$, we get

$$
\begin{aligned}
\left\|S_{n, a}^{\alpha, \beta}\left(t^{2} ; x\right)-x^{2}\right\|_{\rho} & \leq \sup _{0 \leq x<\infty} \frac{\left|S_{n, a}^{\alpha, \beta}\left(t^{2} ; x\right)-x^{2}\right|}{1+x^{2}} \\
& \leq\left|\frac{n\left(a+2 n+\alpha^{2}+2 a n+n^{2}\right)+\alpha(n-1)(2 a+2 n+\alpha)}{(n+\beta)^{2}(n-1)}-1\right| \\
& =\left|\frac{B}{(n+\beta)^{2}(n-1)}\right| .
\end{aligned}
$$

As a result, we obtain

$$
\lim _{n \rightarrow \infty}\left\|S_{n, a}^{\alpha, \beta}\left(t^{v} ; x\right)-x^{v}\right\|_{\rho}=0, \quad v=0,1,2 .
$$

Thus, the proof is completed. 
Theorem 5.3. Let $x \in(0, \infty), n \in \mathbb{N}$ and $f \in C_{B}$. For the operators

$$
S_{n, a}^{\alpha, \beta}(f ; x)=e^{-\frac{a x}{1+x}} \sum_{k=0}^{\infty} \frac{P_{k}(n, a)}{k !} \frac{x^{k}}{(1+x)^{n+k}} \frac{x^{n+1}}{n !} \int_{0}^{\infty} y^{n} e^{-x y} f\left(\frac{\frac{k n}{x y}+\alpha}{n+\beta}\right) d y
$$

and

$$
L_{n, a}^{\alpha, \beta}(f ; x)=e^{-\frac{a x}{1+x}} \sum_{k=0}^{\infty} \frac{P_{k}(n, a)}{k !} \frac{x^{k}}{(1+x)^{n+k}} f\left(\frac{k+\alpha}{n+\beta}\right),
$$

the inequality

$$
\left|S_{n, a}^{\alpha, \beta}(f ; x)-L_{n, a}^{\alpha, \beta}(f ; x)\right| \leq w(f ; \delta) \varphi(x)
$$

is holds true, where

$$
\varphi(x)=\left(1+\frac{1}{\delta} \sqrt{\frac{n(n+1)+2 a n+a^{2}}{(n-1)(n+\beta)^{2}} x^{2}+\frac{n+a}{(n-1)(n+\beta)^{2}} x}\right)
$$

and

$$
\delta=\sqrt{\frac{n(n+1)+2 a n+a^{2}}{(n-1)(n+\beta)^{2}} x^{2}+\frac{n+a}{(n-1)(n+\beta)^{2}} x} .
$$

Proof. From the definition and properties of modulus of continuity, we have

$$
\begin{aligned}
& \left|S_{n, a}^{\alpha, \beta}(f ; x)-L_{n, a}^{\alpha, \beta}(f ; x)\right| \\
& \leq e^{-\frac{a x}{1+x}} \sum_{k=0}^{\infty} \frac{P_{k}(n, a)}{k !} \frac{x^{k}}{(1+x)^{n+k}} \frac{x^{n+1}}{n !} \int_{0}^{\infty} y^{n} e^{-x y}\left|f\left(\frac{\frac{k n}{x y}+\alpha}{n+\beta}\right)-f\left(\frac{k+\alpha}{n+\beta}\right)\right| d y \\
& \leq w(f, \delta)+\frac{1}{\delta} w(f, \delta) e^{-\frac{a x}{1+x}} \sum_{k=0}^{\infty} \frac{P_{k}(n, a)}{k !} \frac{x^{k}}{(1+x)^{n+k}} \frac{x^{n+1}}{n !}\left[\int_{0}^{\infty} y^{n} e^{-x y}\left|\frac{\frac{k n}{x y}+\alpha}{n+\beta}-\frac{k+\alpha}{n+\beta}\right|\right] d y .
\end{aligned}
$$

By applying the Cauchy-Schwarz inequality two times succesively to the right side, we get

$$
\begin{aligned}
& \left|S_{n, a}^{\alpha, \beta}(f ; x)-L_{n, a}^{\alpha, \beta}(f ; x)\right| \\
& \leq w(f, \delta)+\frac{1}{\delta} w(f, \delta)\left\{\left(e^{-\frac{a x}{1+x}} \sum_{k=0}^{\infty} \frac{P_{k}(n, a)}{k !} \frac{x^{k}}{(1+x)^{n+k}} \frac{x^{n+1}}{n !} \int_{0}^{\infty} y^{n} e^{-x y}\left(\frac{\frac{k n}{x y}+\alpha}{n+\beta}-\frac{k+\alpha}{n+\beta}\right)^{2} d y\right)^{\frac{1}{2}}\right. \\
& \left.\times\left(e^{-\frac{a x}{1+x}} \sum_{k=0}^{\infty} \frac{P_{k}(n, a)}{k !} \frac{x^{k}}{(1+x)^{n+k}} \frac{x^{n+1}}{n !} \int_{0}^{\infty} y^{n} e^{-x y} d y\right)^{\frac{1}{2}}\right\} \\
& =w(f, \delta)+\frac{1}{\delta} w(f, \delta) \sqrt{S_{n, a}^{\alpha, \beta}\left(\left(\frac{\frac{k n}{x y}+\alpha}{n+\beta}-\frac{k+\alpha}{n+\beta}\right)^{2} ; x\right)} .
\end{aligned}
$$


If we calculate the $S_{n, a}^{\alpha, \beta}\left(\left(\frac{\frac{k n}{x y}+\alpha}{n+\beta}-\frac{k+\alpha}{n+\beta}\right)^{2} ; x\right)$, we show that

$$
\begin{aligned}
& S_{n, a}^{\alpha, \beta}\left(\left(\frac{\frac{k n}{x y}+\alpha}{n+\beta}-\frac{k+\alpha}{n+\beta}\right)^{2} ; x\right)=S_{n, a}^{\alpha, \beta}\left(\frac{k^{2}\left(\frac{n}{x y}-1\right)^{2}}{(n+\beta)^{2}} ; x\right) \\
& =\frac{1}{(n-1)(n+\beta)^{2}}\left[\left(n(n+1) x^{2}+2 a n \frac{x^{2}}{(1+x)}+\frac{a^{2} x^{2}}{(1+x)}+n x+\frac{a x}{(1+x)}\right)\right] \\
& \leq \frac{n(n+1)+2 a n+a^{2}}{(n-1)(n+\beta)^{2}} x^{2}+\frac{n+a}{(n-1)(n+\beta)^{2}} x,
\end{aligned}
$$

from which, it follows

$$
\lim _{n \rightarrow \infty} S_{n, a}^{\alpha, \beta}\left(\left(\frac{\frac{k n}{x y}+\alpha}{n+\beta}-\frac{k+\alpha}{n+\beta}\right)^{2} ; x\right)=0 .
$$

Thus, we have

$$
\begin{aligned}
& \left|S_{n, a}^{\alpha, \beta}(f ; x)-L_{n, a}^{\alpha, \beta}(f ; x)\right| \\
\leq & w(f, \delta)+\frac{1}{\delta} w(f, \delta) \sqrt{\frac{n(n+1)+2 a n+a^{2}}{(n-1)(n+\beta)^{2}} x^{2}+\frac{n+a}{(n-1)(n+\beta)^{2}}} x \\
\leq & w(f, \delta) \varphi(x) .
\end{aligned}
$$

\section{Voronovskaya Type Theorem}

Lemma 6.1. For the operators $S_{n, a}^{\alpha, \beta}(f ; x)$ defined (2.1), we have

$$
\begin{aligned}
& S_{n, a}^{\alpha, \beta}(t-x ; x)=\frac{\alpha-\beta x}{n+\beta}+\frac{a x}{(n+\beta)(1+x)} . \\
& \begin{aligned}
S_{n, a}^{\alpha, \beta}\left((t-x)^{2} ; x\right)= & \frac{2 n^{2}+n \beta^{2}-\beta^{2}}{(n+\beta)^{2}(n-1)} x^{2}+\frac{2 a(n+\beta-n \beta)}{(n+\beta)^{2}(n-1)} \frac{x^{2}}{1+x}+\frac{a^{2} n}{(n+\beta)^{2}(n-1)} \frac{x^{2}}{(1+x)^{2}} \\
& +\frac{\left(n^{2}-2 \alpha \beta n+2 \alpha \beta\right)}{(n+\beta)^{2}(n-1)} x+\frac{a n+2 a \alpha(n-1)}{(n+\beta)^{2}(n-1)} \frac{x}{1+x}+\frac{\alpha^{2}}{(n+\beta)^{2}} .
\end{aligned}
\end{aligned}
$$

Proof. By using the definition of $S_{n, a}^{\alpha, \beta}$, it can be proved easily.

Theorem 6.1. Let $a, x>0,0 \leq \alpha \leq \beta$ and $n \in N$. For $f \in C^{2}(0, \infty)$ and bounded, we have

$$
\lim _{n \rightarrow \infty}(n+\beta)\left[S_{n, a}^{\alpha, \beta}(f ; x)-f(x)\right]=\left(\alpha-\beta x+\frac{a x}{1+x}\right) f^{\prime}(x)+\frac{2 x^{2}+x}{2} f^{\prime \prime}(x) .
$$


Proof. Let $x, t \in(0, \infty), f \in C^{2}(0, \infty)$. By Taylor's formula for $f$, we have

$$
f(t)=f(x)+(t-x) f^{\prime}(x)+\frac{(t-x)^{2}}{2 !} f^{\prime \prime}(x)+(t-x)^{2} \phi(t ; x)
$$

where the function $\phi(t ; x) \in C[0, \infty)$ and $\lim _{t \rightarrow x} \phi(t ; x)=0$. By applying the operator $S_{n, a}^{\alpha, \beta}$ to the both sides of (6.1), we have

$$
\begin{aligned}
S_{n, a}^{\alpha, \beta} f(t)= & f(x) S_{n, a}^{\alpha, \beta}(1 ; x)+f^{\prime}(x) S_{n, a}^{\alpha, \beta}(t-x ; x)+\frac{f^{\prime \prime}(x)}{2 !} S_{n, a}^{\alpha, \beta}\left((t-x)^{2} ; x\right) \\
& +S_{n, a}^{\alpha, \beta}\left((t-x)^{2} \phi(t ; x) ; x\right) .
\end{aligned}
$$

According to Lemma6.1, the equality (6.2) can be written as follows

$$
\begin{aligned}
& (n+\beta)\left[S_{n, a}^{\alpha, \beta}(f ; x)-f(x)\right]=(n+\beta)\left[\frac{\alpha-\beta x}{n+\beta}+\frac{a x}{(n+\beta)(1+x)}\right] f^{\prime}(x) \\
& +(n+\beta)\left[\frac{2 n^{2}+n \beta^{2}-\beta^{2}}{(n+\beta)^{2}(n-1)} x^{2}+\frac{2 a(n+\beta-\beta \beta)}{(n+\beta)^{2}(n-1)} \frac{x^{2}}{1+x}+\frac{a^{2} n}{(n+\beta)^{2}(n-1)} \frac{x^{2}}{(1+x)^{2}}\right. \\
& \left.+\frac{\left(n^{2}-2 \alpha \beta n+2 \alpha \beta\right)}{(n+\beta)^{2}(n-1)} x+\frac{a n+2 a \alpha(n-1)}{(n+\beta)^{2}(n-1)} \frac{x}{1+x}+\frac{\alpha^{2}}{(n+\beta)^{2}}\right] \frac{f^{\prime \prime}(x)}{2 !}+S_{n, a}^{\alpha, \beta}\left((t-x)^{2} \phi(t ; x) ; x\right),
\end{aligned}
$$

where

$$
\begin{aligned}
& S_{n, a}^{\alpha, \beta}\left((t-x)^{2} \phi(t ; x) ; x\right)= \\
& =e^{-\frac{a x}{1+x}} \sum_{k=0}^{\infty} \frac{P_{k}(n, a)}{k !} \frac{x^{k}}{(1+x)^{n+k}} \frac{x^{n+1}}{n !} \int_{0}^{\infty} y^{n} e^{-x y} f\left(\frac{\frac{k n}{x y}+\alpha}{n+\beta}-x\right)^{2} \phi(t ; x) d y .
\end{aligned}
$$

By applying the Cauchy-Schwarz inequality two times succesively to the right side, we get

$$
(n+\beta) S_{n, a}^{\alpha, \beta}\left((t-x)^{2} \phi(t ; x) ; x\right) \leq \sqrt{(n+\beta)^{2} S_{n, a}^{\alpha, \beta}\left((t-x)^{4} ; x\right)} \sqrt{S_{n, a}^{\alpha, \beta}\left(\phi^{2}(t ; x) ; x\right)} .
$$

From Lemma 6.1, we have $S_{n, a}^{\alpha, \beta}\left((t-x)^{4} ; x\right)=O\left(n^{-2}\right)$. Thus, we get

$$
\lim _{n \rightarrow \infty}(n+\beta)^{2} S_{n, a}^{\alpha, \beta}\left((t-x)^{4} ; x\right)=12 x^{4}+12 x^{3}+3 x^{2} .
$$

On the other hand, since $\phi(t ; x) \in C[0, \infty)$ and $\lim _{t \rightarrow x} \phi(t ; x)=0$, then we conclude

$$
\lim _{n \rightarrow \infty} S_{n, a}^{\alpha, \beta}\left(\phi^{2}(t ; x) ; x\right)=\phi^{2}(x ; x)=0 .
$$


Hence, we get from (6.3), (6.4) and (6.5) that

$$
\lim _{n \rightarrow \infty}(n+\beta) S_{n, a}^{\alpha, \beta}\left((t-x)^{2} \phi(t ; x) ; x\right)=0
$$

and then, we find

$\lim _{n \rightarrow \infty}(n+\beta)\left[S_{n, a}^{\alpha, \beta}(f ; x)-f(x)\right]=\left(\alpha-\beta x+\frac{a x}{1+x}\right) f^{\prime}(x)+\frac{2 x^{2}+x}{2} f^{\prime \prime}(x)$

which completed the proof. [1]

\section{RE F E R E N C E S}

1. A. Erencin, B. Sevim, A modification of generalized Baskakov Kantorovich operators, Stud. Univ. Babeş-Bolyai Math.59 (2014), 3, 351-364.

2. A.Erencin, G. Bascanbaz-Tunca, Approximation properties of a class of linear positive operators in weighted spaces, C.R. Acad. Bulgare Sci., 63(2010), no. 10, 1397-1404.

3. A. Erencin, Durrmeyer type modification of generalized Baskakov operators, Appl. Math. Comput. 218 (2011) 4384-4390.

4. A.D.Gadjiev, Theorems of the type of P. P. Korovkin's theorems, Math. Zametki, 20 (1976), no. 5-6, 781-786(in Russian), Math. Notes, 20(1976), no. 5-6, 995-998 (Endl. Trans.)

5. A. Kajla, On the Bézier Variant of the Srivastava-Gupta Operators, Constr. Math. Anal.,(2)(2018), 99-107

6. A.Wafi, S. Khatoon, The Voronovskaya theorem for generalized Baskakov-Kantorovich operators in polynomial weight spaces, Mat. Vesnik, 57(2005), no. 3-4, 87-94.

7. A. Lupas, and M. Müller, Approximationseigenschaften der Gammaoperatoren, Math. Zeitschr. 98(1967), 208-226.

8. A. Wafi, N. Rao, Stancu-Variant of Generalized Baskakov Operators, Filomat 31(2015) (9), 2625-2632.

9. E. Pandey, R.K. Mishra, S.P. Pandey, Approximation Properties of Some Modified Summation-Integral Type Operator, (IJSCE) ISSN: , Vol.5 Issue-1, (2015), 2231-2307.

10. F.L. Cao, C.M. Ding, Lp approximation by multivariate Baskakov-Kantorovich operators, J. Math. Anal. 27 (1978), pp. 127-142.

11. F.L. Cao, C.M. Ding, Z.B. Xu, On multivariate Baskakov operator, J. Math. Anal. Appl. 348 (2008) 856-861.

12. H. G. İnce İlarslan, T. Acar, Approximation by bivariate (p,q)-Baskakov-Kantorovich operators, Georgian Mathematical Journal, 25 (3), 2018, 397-407.

13. I. Krech, R. Malejki.Approximation of functions of several variables by the BaskakovDurrmeyer type operators, Technical Transactions FundamentalSciences.(2016) DOI:2353737XCT.16.142.5753, 98-105 
14. L. Rempulska, M. Skorupka, Approximation properties of modified gamma operators, Integral Transforms and Special Functions, Vol.18, No. 9, (2007), 653-662.

15. M. Goyal, P. N. Agrawal, Bivariate generalized Baskakov-Kantorovich operators, (2015)arXiv preprint arXiv:1505.06071

16. M. Bodur, O.G. Yılmaz, A. Aral, Approximation by Baskakov-Szasz-Stancu Operators Preserving Exponential Functions, Constr. Math. Anal., 1 (1) (2018), 88-98

17. M. Murselan, M. Nasurizzaman, Approximation of Modified Jakimovski- LeviatanBeta type operators, Constr. Math. Anal. 1 (2) (2018) 88-98

18. R. Malejki, E. Wachnicki, On the Baskakov-Durrmeyer type operators, Comment. Math. 54.1(2014), 39-49.

19. S. N. Deveci, T. Acar, O. Alagöz, Approximation by Gamma type Operators, Mathematical Methods in the Pplied Sciences, 43 (5), (2020), 2772-2782

20. S.Guo, Q.Qiulan, On pointwise estimate for gamma operators. Approximation Theory and its Applications, (2002), 18(3): 93-98.

21. T. Acar, V. Gupta and A. Aral, Rate of Convergence for Generalized Szasz Operators, Bulletin of Mathematical Science, 1 (1), 99-113 (2011)

22. V.A. Baskakov, An example of a sequence of linear positive operators in the spaces of continuous functions, Dokl. Acad. Nauk SSSR 113 (1957) 249-251

23. V.Totik, The gamma operators in Lp spaces. Publicationes Mathematicae (Debrecen), (1985), 32: 43-55.

24. V. Mihesan, Uniform approximation with positive linear operators generated by generalized Baskakov method, Au-tomat. Comput. Appl. Math. 7 (1998) 34-37.

25. Z. Walczak, Baskakov type operators, Rocky Mountain J. Math., 39 (3) (2009), pp. 981-993.

26. Z. Walczak, V. Gupta, A note on the convergence of Baskakov type operators, Appl. Math. Comput. 202 (2008), no. 1, 370-375. 